\title{
AN INVESTIGATION OF A GENERATIVE PARAMETRIC DESIGN APPROACH FOR A ROBUST SOLUTION DEVELOPMENT
}

\author{
H. Li ${ }^{凶}$, T. Brockmöller, P. C. Gembarski and R. Lachmayer \\ Leibniz Universität Hannover, Germany \\ $\triangle$ li@ipeg.uni-hannover.de
}

\begin{abstract}
The main design activity in engineering practice is to adapt existing designs or to create variants of existing products to new demands, which require a robust model against both parametric and topological changes. To design such a kind of model becomes a big challenge, especially in the development of structural components due to the number of load application points, variable load cases and restrictions from manufacturing technologies. Thus, the generative parametric design approach is applied to generating high dynamic product models, allowing them to be adjusted for changes feasibly.
\end{abstract}

Keywords: computer-aided design (CAD), knowledge-based engineering (KBE), product development, generative parametric design approach

\section{Introduction}

The main design activity of designers during the product development process is to adapt existing designs or to create variants of existing products to new demands. Original designs represent only a small part. In the case of the design task is adaptive or variant design, already known solution principles are retained, but the components are modified to new requirements (Pahl et al., 2013). This type of design often involves routine activities since the basic structure of the components remains unchanged (Stokes, 2001). Particularly in the case of intergenerational product development, such as technical inheritance, the design process requires a robust model for the adaption by considering the assembled and verified usage information of the product life cycle from one generation to the next (Mozgova et al., 2015; Demminger et al., 2016). Thus, it is advantageous to model both simple and complex components and assemblies as highly dynamic product models in CAD- systems.

\subsection{Motivation}

The challenge is to build complex product models in such a way that they are robust against both parametric and topological changes ( $\mathrm{Li}$ and Lachmayer, 2019). Robustness refers to errors or instability problems that can be caused by changes in the geometric model (Amadori et al., 2012). This requires a model structure in which the dependencies of the model parameters are locally limited and there are as few global dependencies as possible. In face of this challenge, several methods have been proposed, e.g., enhancing the generation of explicit models by parametric design (Kim and Han, 2007); master model creation and manipulation by feature-based modeling (Yin and Ma, 2012) and knowledge-based application development (Boyle et al., 2011; Hirz et al., 2013). These models are in some case hard to 
adapt themselves to new variants because the required adaptations are way too large to be mapped into one single model due to the lack of knowledge and the evolving requirements (Gembarski et al., 2017). Particularly it becomes a big challenge in the development of structural components because of the number of load application points, variable load cases and geometrical restrictions from available manufacturing technologies. In this sense, a new modeling approach is needed, enabling the flexible generation of design variations without errors and minimum effort. Thus, the following article describes an approach for generating highly dynamic product models for complex components and assemblies by combining the advantages of parametric, knowledge-based and generative modeling methods.

\subsection{Paper structure}

The structure of the remaining paper is reflected as follows: the main modeling approaches in current use and their limitations in robust model development, are discussed in section 2. Based on the problems identified in section 2, the generative parametric design approach (GDPA) is in detailed introduced in section 3, in which the basic idea and its structure are clarified. In section 4, GDPA is implemented to create a vehicle door frame following certain design procedures. Section 5 provides a short discussion in which the advantages and limitations of GPDA are presented. Finally, the associated conclusions and an outlook of future researches are given.

\section{Review of CAD modeling approaches}

Since there may potentially be an infinite number of possible solutions to a given problem, CAD models should provide a clear structure to capture the understanding of the problem and represent the feasible solutions. In this context, the CAD modeling approaches have to provide support to facilitate the generation of robust CAD models. Thus, the following sections contribute to obtaining an overview of the main modeling approaches in current use.

\subsection{Parametric design}

Parametric design (PD) means the use of a CAD system to automatically modify a design as the values of the parameter change and to make corresponding changes to the CAD model during the design process (Hirz et al., 2013). Given a design problem, the requirements are transformed into a set of parameters and their constraints are also represented via a series of mathematical or logical relations in a hierarchy manner, capturing the product's behaviors and the structures (Vajna et al., 2009). As the design progresses further, parameters are introduced describing the dimensions of the object and potentially physical properties to be used for its construction. Therefore, PD offers potentials in quickly design solution generation in terms of the requirements and also maintains the design history steps to create variable CAD models instead of keeping only the one outcome model (Hoffmann, 2005).

However, since only the explicit interdependencies between parameters as well as the initial predefined constraints can be used for change adaption, the designers are required to structure the design operations at the beginning of the design process. So, a parametric model offers little flexibility once the parameters and their interdependencies have been decided. Thus, the generation of variations with such dependency chains means that the designer must previously examine the design task to define which parts are to be represented and to determine the sequence of operations needed to create these parts. This modeling logic is sometimes visible in auxiliary geometric elements present in the model or in the layers that organize the model but, in most cases, it is "rarely recoverable and difficult to decipher" (Park and Holt, 2010). This means that designers often attempt to generate design variations in the absence of a clear appreciation of the actual reasoning underlying the design, as very little modeling logic can be reused.

\subsection{Knowledge-based design}

Knowledge-based design (KBD) is a research area for CAD models generation that involves complex and iterative processes by capture and reuse of engineering knowledge (VDI, 2017). The objective of KBD is the reduction of time and cost of product development by means of: (1) 
Automation of repetitive, non-creative design tasks; (2) Support of multidisciplinary design optimization in all phases of the design process (La Rocca, 2011). Once engineering knowledge about the problem is collected and stored as a generic knowledge-based CAD, designers can generate and evaluate different design variations quickly and easily by changing the input specifications for the model or modify designs by extending or changing the model. This means that the generic knowledge-based CAD models can be used repeatedly to rapidly develop design variations in a fully associative and automatic way.

However, the formalized engineering knowledge is too often encapsulated in CAD models and inference engines of KBD applications, which are not easily accessible for designers. Thus, reuse of the formalized knowledge requires additional effort, becomes a serious limitation (Verhagen et al., 2012). Further, the knowledge base is normally task-based towards a black-box because the formalized knowledge is not explained and contextualized, which leads to a problem that the solution variations will be generated in association with increasing efforts (Cho et al., 2016).

\subsection{Generative design}

Generative design (GD) is an iterative approach in which the exploration of a new design is deemed as a series of transformations from an early initial data to a large number of possibilities of knowledge integrated designs, which have high qualities that meet the design requirements in the best possible way (Cui and Tang, 2017). GD leverages production rules drawn from existing engineering knowledge to autonomously generate a high number of solution alternatives, corresponding to the specific requirements and restrictions, e.g., target weight and stress. The GD design process is normally starting with the representation of design ideas via a set of algorithmic rules. After that, generative mechanisms allow the computer to generate the design solutions automatically and visualize the design solutions as outputs for evaluation by designers (Krish, 2011). GD is used for various purposes, therefore several methods have been successfully developed during the last two decades, in which the most used methods to CAD models creation are graph grammar and spatial grammar, as they have the potential to create structural components that can be directly used in a CAD environment (Chakrabarti et al., 2011). The former uses a vocabulary of graph and a set of rules to form CAD model through a network of graphs. The set of rules used for this purpose has mechanisms that can identify and modify defined structures within the graph network. If a rule is used, the structure of the graph network is first searched for and then replaced by a structure that is usually defined (Helms, 2013). Spatial grammar uses a 3D geometry representation instead of the graph network and the vocabulary consists of parametric geometric primitives (Hoisl, 2012).

Due to the variety of geometrical dependencies and the geometric complexity of structural components, this is currently still a challenge to formulate the engineering knowledge via production rules (Sauthoff, 2017). Although CAD models can in principle be represented by design languages, the questions of that how many rules are needed and how these rules and the vocabulary can be developed based on existing knowledge, are not yet clear (Gips, 1999). Further, due to the domain and process knowledge are not formulated in an explicit manner associated with a specific geometric model, a significant effort is expected by adapting the set of the production rules. Otherwise, it is hard to guarantee that the solution generated through such rules possesses the required product properties.

\section{Generative parametric design approach}

\subsection{Idea of GPDA}

In the course of research into technical inheritance, a modeling strategy was needed for, with which the changing requirements of a generation change of a product can be automatically incorporated into the associated CAD model in the form of a shape change (Lachmayer et al., 2017). This requires a modeling strategy to create highly dynamic product models. The challenge is that the models must be adaptable both parametrically and topologically so that the best possible solution can be generated. 
This requires robust models that guarantee reconstruction after changes. This in turn assumes that the dependencies of the individual model parameters must be reduced or locally limited. An optimal solution also requires that all necessary information for manufacturing restrictions are also taken into account so that a model close to the final geometry can be created.

A purely parameter-based approach is insufficient. The implementation of design knowledge is necessary to be able to conclude the design characteristics from the product properties specified by the requirements. However, a purely knowledge-based approach also has disadvantages, since complex models have a large number of dependencies and are rigid in their structure. Generative design offers the possibility to create virtually any geometry through the use of grammars and a set of production rules to expand the solution space considered. Nevertheless, the disadvantage is that a geometry close to the final geometry and thus the consideration of manufacturing restrictions is only possible with considerable effort. For example, after a topology optimization, the component shape must always be subsequently adapted. Therefore, this paper proposes an approach for the creation of CAD models that combines generative with a parametric knowledge-based modeling strategy. This allows a large solution space to be spanned for parametric models.

The GPDA follows the idea of reducing the global dependencies in the model, as these limit flexibility. It is possible, that a change in geometry can lead to the loss of dependencies and the inability to rebuild the models. To achieve this, the model structure is separated from the product structure of a component or assembly. This means that individual components no longer have to be a single CAD model, but can be a combination of several. In assemblies, the interfaces of the individual components are no longer where they are located in a physically existing product, but where the smallest dependencies for the overall model arise.

Figure 1 shows the connection of a hollow profile with a plate. Depending on the application, the connection between the two components can be made using screws or a welded joint. In the physically existing product, the connection is between the two components plate and hollow profile. In the CAD model, the connection of the components is defined in a so-called design element. This design element has several interfaces that connect it to the overall model. These are located in a square section of the plate and at the other end of the hollow profile. This design element can now be replaced in its entirety by another design element which, for example, has a different type of connection, provided that it has the same parameter set in order to be able to communicate with the interfaces. The advantage of this is that the CAD model structure does not change. If the interface were directly in the connection between the plate and the hollow profile, the model structure would be different with each change due to the topological differences of the components and the number of connecting elements. As a result, more dependencies have to be defined in the model and the effort required to create a robust model increases.
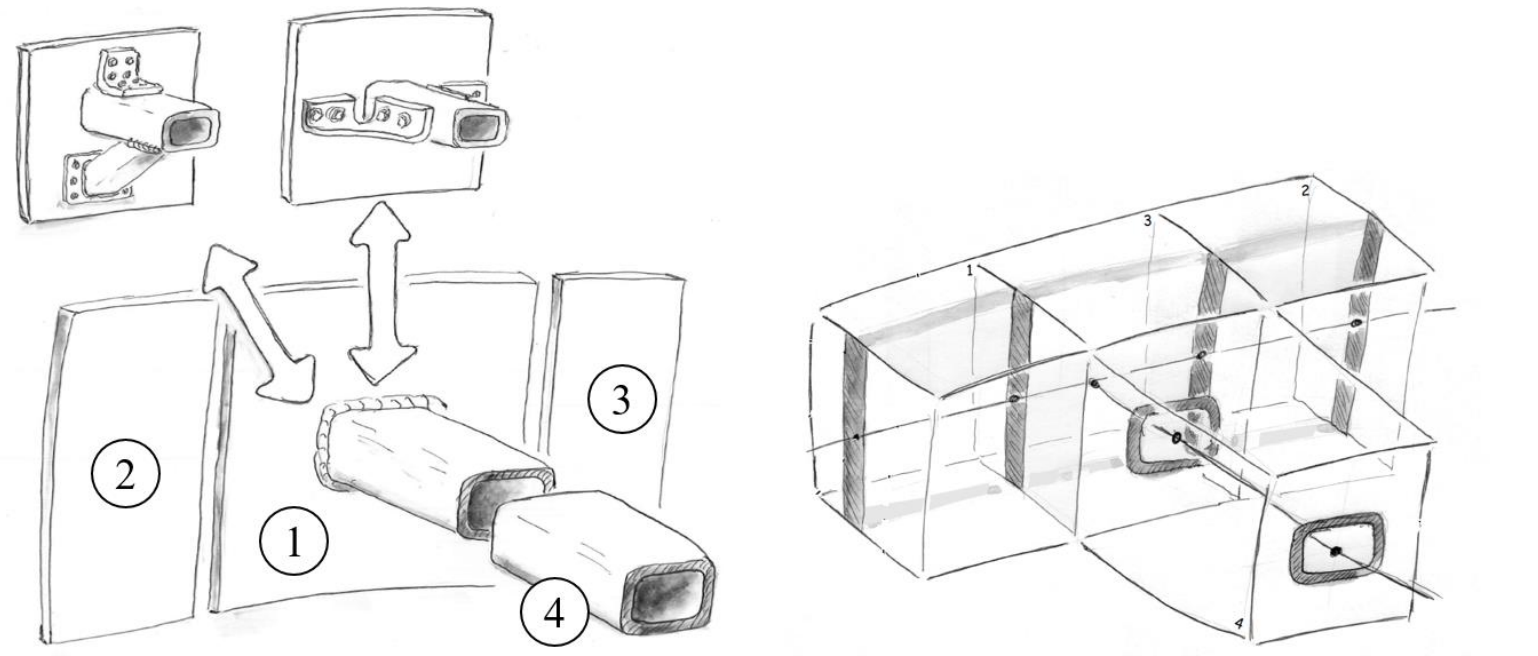

Figure 1. Left: Principle GPDA model; right: Model structure with skeleton and interfaces (acc. to Sauthoff, 2017) 


\subsection{Structure of the GPDA}

\subsubsection{Design zone}

Design zones are placeholders for design elements that represent solutions for each design zone. The essential characteristic of a design zone is the definition of the number and arrangement of reference interfaces, wherein a reference geometry in the form of a flat or curved surface is determined. In order to ensure a continuous geometry transition between design elements within a model, neighboring design zones are interconnected by a reference interface.

\subsubsection{Design skeleton}

The design skeleton is a parametric model that is defined on the basis of points, lines and planes. Such a skeleton provides a parametric description of the dimensions of the design zones as well as the positions and orientations of the reference geometry for the connection interfaces. By defining this design skeleton, the number of interdependencies is decreased to a minimum by separating the modeling structure and assembly structure independently. The skeleton thus represents the highest level in the dependence chain, which is why its geometric stability must be ensured in any case.

\subsubsection{Connection interfaces}

The connection interfaces are two-dimensional sketches that determine the transition geometry from one design element to another, consisting of a geometrical profile and the parametric description of the profile itself. The connection interfaces are located in the reference interfaces between design zones so that their positions are determined by the skeleton. The geometry of the sketches is determined by superordinate reference parameters. A library containing different variants is required so that different interface geometries can be taken into account. In the case of the example from Figure 1, this can be a circular hollow profile instead of a rectangular one.

\subsubsection{Design elements}

Design elements are designed as identifiable constructive solutions for each design zone enriched with engineering knowledge, which are the essential building blocks of the GPDA. The more design elements are available for a design zone in a library, the wider the available solution space can be spanned. In order to ensure a consistent transition to the neighboring elements, the design elements at the reference interfaces are controlled by the connection interfaces. The modeling structure of the design element and the respective design zone are identical. The design element has the same skeletal structure and the same type of interfaces. The parameters for the design elements and the connection interfaces are linked to the design skeleton. Depending on how the structure of the overall model changes, the design elements change accordingly.

\subsection{Optimization of the geometry}

A computer-aided engineering environment is required so that an optimized geometry can be generated automatically with a model that has a GPDA model structure proposed above. This iteratively adapts the component geometry by alternating synthesis and analysis steps, e.g. with an evolutionary algorithm, until the requirements are met. An evolutionary algorithm has been chosen at this point, as a large number of parameters can be considered in this way. Figure 2 illustrates the proposed sequence of the engineering environment. The central module is the Opti-Toolbox. This VB.NET based program controls the entire engineering environment. Here the user defines the target function for optimization. The algorithm then decides how the CAD model should be configured in the CAD system (here Autodesk Inventor is used). Different variants with different parameter configurations are created in the first generation. The CAD model is transferred to the Abaqus FE system as a STEP file for analysis. The boundary conditions and required calculation information are also transferred to Abaqus from the Opti-Toolbox as an XML file via a Python interface. After the analysis the results of the simulation are interpreted by the Opti-Toolbox and it is checked whether one of the variants of the first generation fulfills the requirements. If this is the case, the optimization 
is completed. If this is not the case, a new generation of variants is created by the algorithm. This is repeated until all requirements are met or a given number of iterations is reached.

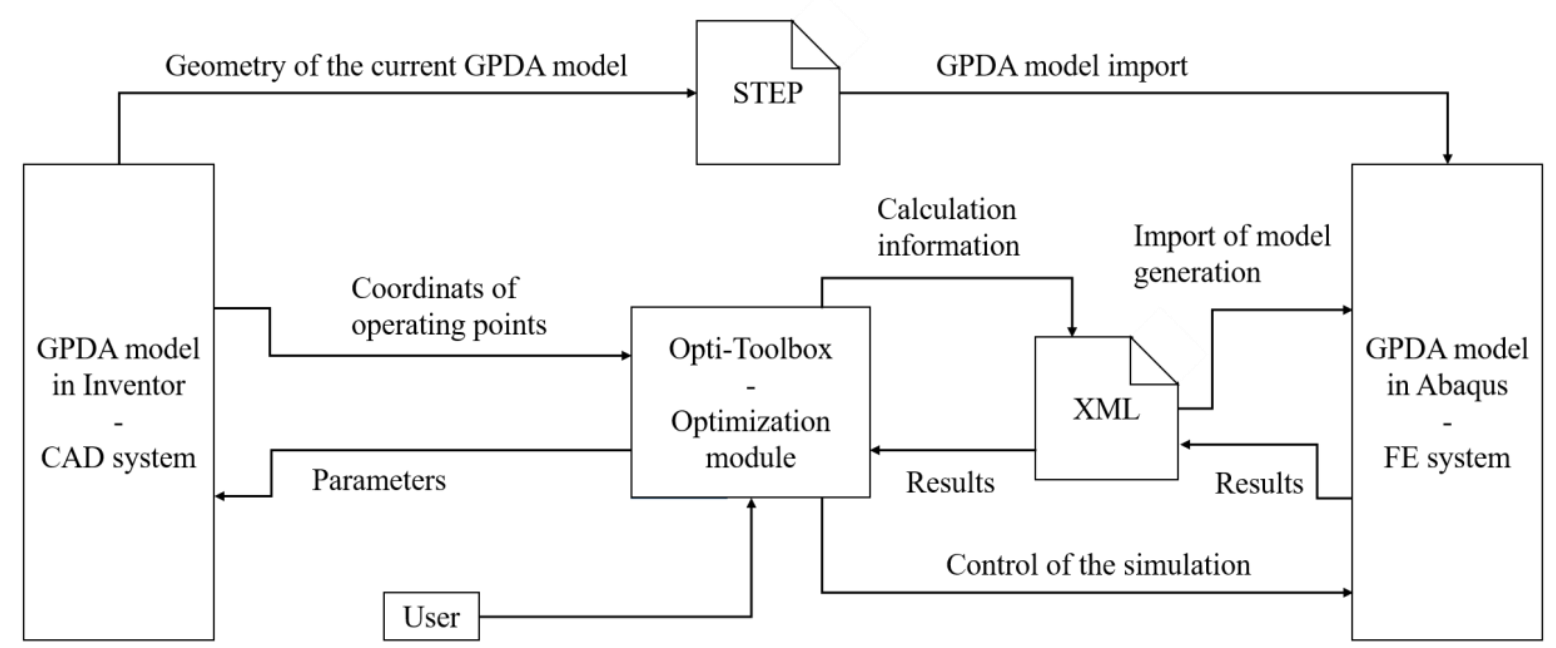

Figure 2. Computer-aided engineering environment for the optimization of GPDA models

\section{Case study}

The previous section identified the capacity that GPDA is able to generate robust CAD models of structural component. For a better understanding of the design process, a vehicle door frame was chosen as an attempt to demonstrate the key points of GPDA implementation.

\subsection{Door design requirements}

Vehicle doors are the key moving parts of a vehicle body that not only allow the passenger engagement with the vehicle appropriately and comfortably, but also provide key structural supports to the vehicle body so that protect the occupants from any impact during a crash situation (Leinweber, 2014). Thus, the development of a vehicle door has to fulfill lots of requirements from variable aspects, e.g., regulation (e.g., safety requirements due to frontal and side impact), packaging (e.g., ergonomic requirements) and manufacturing (e.g., material and manufacturing techniques). Regarding the door frame design, special attention has to be paid to the stiffness requirements, since the door must withstand various loads without affecting the operational stability. Based on the experience from design practices, four typical load cases need to consider simultaneously during design, as depicted in Figure 3 (Morello et al. 2011).
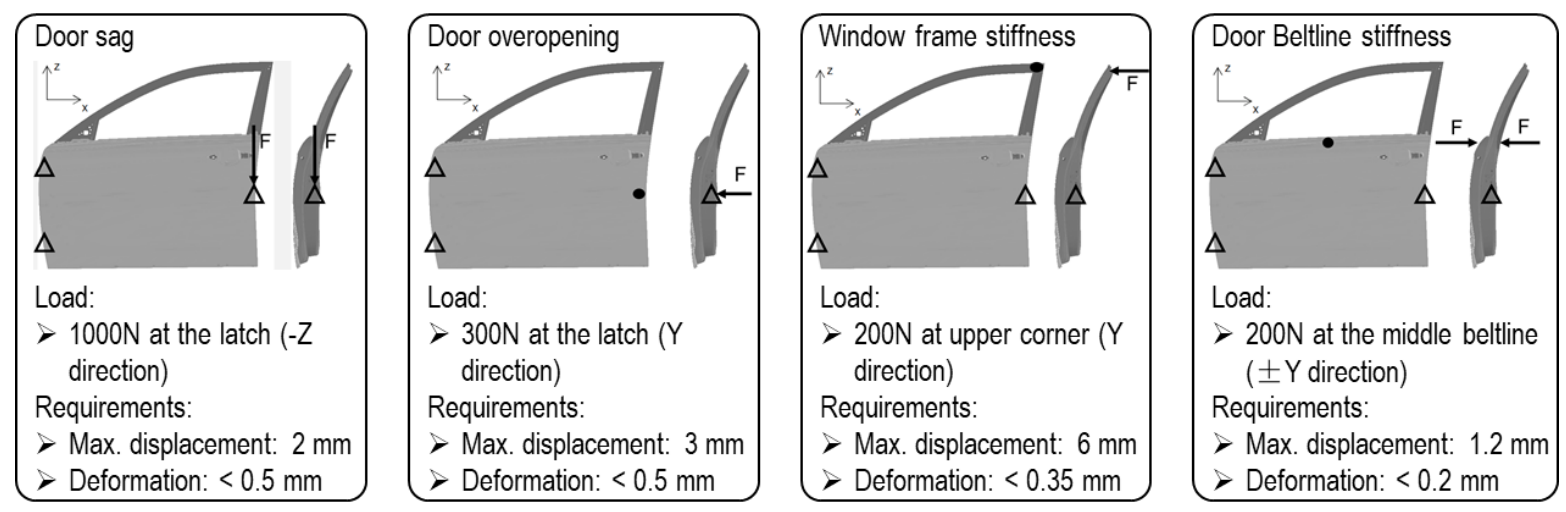

Figure 3. Structural requirements of vehicle door frame design (acc. to Morello et al. (2011))

However, the specifications of such load cases of door design vary from one product to another as well as one generation to the next. Since the definitions of the load cases keep the same, the development of next 
generation doors or adaptation to new requirements is deemed to be the routine work, which is a time consuming and costly task (Tabrizi and Walleigh, 1997). From this point of view, a robust CAD door model with high flexibility and robustness enables designers to reflect such changes quickly, which is needed.

\subsection{GPDA-based door robust solution development}

In the context of GPDA design process, in addition to the design requirement as well as the boundary conditions of door development, a structural design is performed in order to determine the effects of loads on door physical structure. Given the results from the structural design, the door is separated into eleven design zones, in which they can be categorized into five joint zones (black regions) and six beam zones (grey regions), as depicted in Figure 4 (left). Consequently, a design skeleton consists of seventeen local coordinate systems is created. Each of the coordinate systems has six spatial degrees-of-freedom, defining the dimensions of the design zones and the positions and orientations of the reference interfaces for locating the connection interfaces (Figure 4, middle). Based on this skeleton, each design zone of the door has a unique modeling structure independent to the assembly structure, which is already linked to neighboring design zones. After that, a connection interface library contains a variety of parametric geometrical profiles is defined, in which the geometrical restrictions come from different manufacturing techniques are implemented and described via a set of parameters or design rules. Three connection interfaces of the window frame beam zone are presented in Figure 4 (middle).

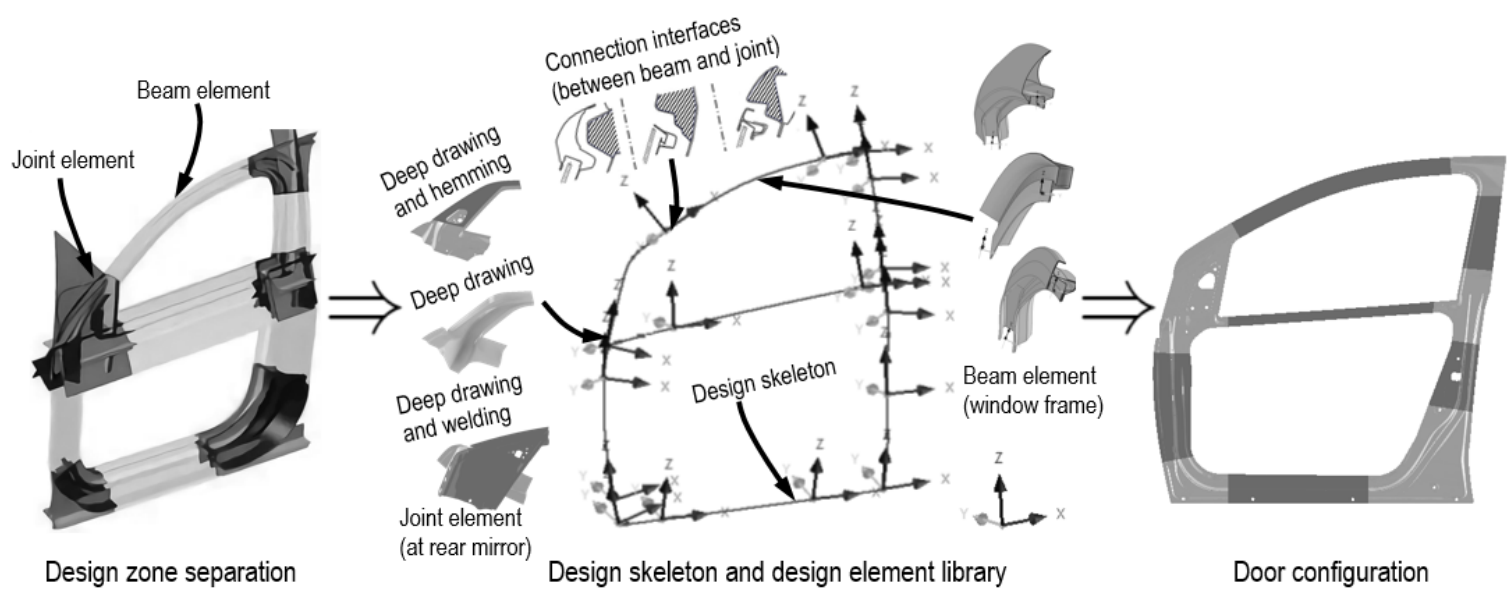

Figure 4. Door development process via GDA and its key elements

Once the connection interfaces are defined, the next step is the establishment of the design element library, making up different geometrical partial models. As seen in Figure 4 (middle), three design elements of the window frame zone are generated through loft operation in CAD system. In this case, the connection interfaces are sketched in the YZ planes of the local coordinate systems. The curve tangent to both $\mathrm{X}$-axes between the coordinate systems is an approximation curve controlled through points that describe the door contour. By taking this curve as the centerline, a loft feature with the defined connection interfaces is generated that tangent to the $\mathrm{X}$-axes of both local coordinate systems to ensure a continual transition to the neighboring design zones. Since the manufacturing techniques (hemming, welding and deep drawing) bring variable restrictions in design, different design elements are modeled with a set of identical parameters, such as the joint elements as shown in Figure 4 (middle). The determining parameters for these elements are the sheet metal thickness, the depth of deep drawing, the length of the welding flange and the hem radius, to name a few of them. In this way, engineering knowledge is transformed into a series of design rules, constraints or parameters that can be understood by CAD systems. Thus, design elements are then knowledge containers allowing to represent the physical solutions of every design zone.

After completion of the design element library, design elements have to be selected and assembled together in order to form a solution in terms of the design requirements. The arrangement of the design elements can be realized in different ways, e.g., a spreadsheet or a macro. For this case study, the CAD-system Autodesk 
Inventor was adapted and extended by iLogic functionalities since it allows the use of multiple KBDmodelling techniques (Gembarski, 2018). In this application, the iLogic rules behind will retrieve the corresponding design elements and configure those at the right place via the selection of connection interfaces, which is basically done by inheriting parameters of the design skeleton to the design elements. The local coordinate systems in the design skeleton aid for positioning design elements one after another and orient each design element in order to generate a required door. At last, the most relevant design solution is identified towards the design requirements, as seen in Figure 4 (right).

As the requirements may change during the development process (especially in the early design stage), the initial GPDA model is in some cases hard to fulfill the requirements. Thus, GPDA provides high flexibility and robustness to solve this challenge, as the resulting impact on the product is largely limited to a few design zones. Once the changes have been anticipated and covered within the design element library, the formalized knowledge provides adaptability against those changes by either modifying or replacing the design elements or changing the parameters of the design elements themselves. When the changes turn out differently than considered, no feasible solution can be generated. In case of this circumstance, the related knowledge will be collected and new design elements possessing the required functions will be added in the design element library. This ensures the new configured solution fulfills the changed requirements. Meanwhile, more and more information and knowledge are accumulated along with the design process, designers can fix the errors of processes designing and identify the more robust solutions by modifying the design elements resided in the design element library. Using this approach, different door variations have been built in terms of different requirements of door structures as discussed in Morello et al. (2011), as shown in Figure 5.

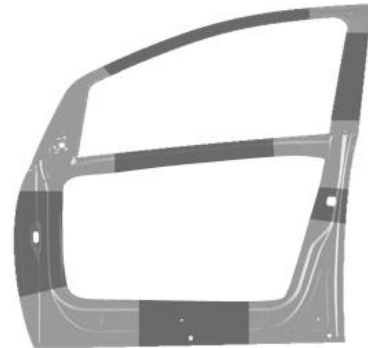

Unitized deep drawn door

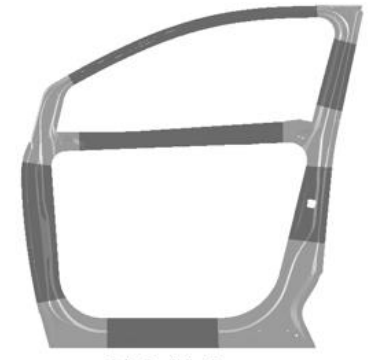

Hybrid door

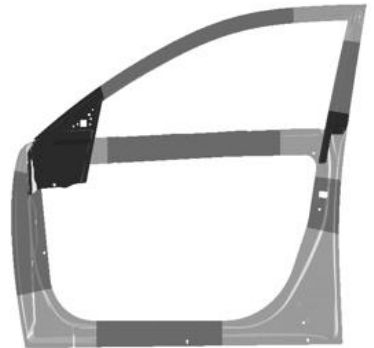

Sash door

Figure 5. Door variations

\section{Discussion}

It is clear from the reviewed literature that different modeling approaches can be applied to achieve robust solutions. However, there was a high degree of agreement that those approaches have also fundamental restrictions or limitations as described in section 2 . The results of the case study suggest that GDPA can overcome the limitations of the aforementioned modeling approaches in developing robust solutions, allowing them to be adjusted for new or changed requirements during the product development process. Three success factors contribute to this achievement.

- Reduction of interdependencies: With the help of a design skeleton, a given problem is performed independently at every design zone. The design elements generated for an individual design zone are therefore kept separate from other design zones as well. Thus, the number of interdependencies are decreased to a minimum by separating the modeling structure and assembly structure independently. As a result, a variation of GPDA model can be executed in both parametrical and topological levels with little effort.

- Integration of engineering knowledge: Within the development process, design elements are considered as design knowledge containers allowing to model the relationships between requirements, functional descriptions and physical solutions of a design zone and are also the vehicle to bring this knowledge to the downstream applications for GPDA model configuration. Such formalized engineering knowledge in each design element enables the generation of robust solutions in a controlled way. 
- Dynamic of the design process: Comparing to the reviewed modeling approaches, the design element library of GPDA keeps dynamic during the development process that the design elements in the library can be added, modified, replaced or even deleted in order to create robust solutions regarding the evolving design requirements. In case the initial GPDA model cannot guarantee a good performance, the appropriate knowledge will be gathered and new design elements with the required functions are added in the design element library. Meanwhile, more information and knowledge are accumulated as the design process progresses. Designers can fix the errors of processes designing and identify the more robust solutions by modifying the design elements involved in the design element library.

The limitations of GDPA should also be noted. It becomes clear that the increasing complexity due to the integration of new and additional functionalities, incorporating multiple engineering domains require a large number of design elements. To build those elements is associated with a considerable effort. Another limitation is due to the fact that design elements map the engineering knowledge discretely, the configured design solutions in sometimes cannot be guaranteed that they represented the best performance to the given design problem. At last, it is absolutely necessary to keep the design element library consistent for robust solution development, calling for an increased effort.

\section{Conclusion and outlook}

This research contributes to investigate GPDA as an effective modeling approach for robust solution development. GPDA permits taking into account the design parameters and uncertainty variables associated with the design of a structural product in forms of design elements as well as the simultaneous integration of manufacturing restrictions during the development process. Once a GPDA model is configured, design changes can be quickly adapted feasibly by either exchanging design elements or modifying design parameters of the design element themselves or design skeleton. Besides, GPDA also makes it simple to examine exactly how design objectives are impacted by design changes, find worthwhile, previously unconsidered design configurations and determine the values of design variables required to solve specific problems. For assessment, meaningful implementation for vehicle door design proved that GPDA provides more concrete aids to overcome the weaknesses observed in design practices at enhancing the diversity and adaptability of the design solutions.

As a recommendation for future research, GPDA can be applied as a collaborative methodology for product development. Since the creation of the design element library requires considerable effort, the separation of design zones permits the parallel or simultaneous accomplishment of the design task by several designers. This will significantly reduce the design effort. Moreover, given the potential of GPDA in the design optimization as described in section 3.3, it is reasonable to assume that an optimized solution can be generated together with FE-analysis and an evolutionary algorithm. The output model is also not only a design solution but also a design concept that the designers could manipulate to handle design changes in the shortest amount of time.

\section{References}

Amadori, K. et al. (2012), "Flexible and robust CAD models for design automation", Advanced Engineering Informatics, Vol. 26 No. 1, pp. 180-195. https://doi.org/10.1016/j.aei.2012.01.004

Boyle, I., Rong, Y. and Brown, D. (2011), "A review and analysis of current computer aided fixture design approaches", Robotics and Computer-Integrated Manufacturing, Vol. 27 No. 1, pp. 1-12. https://doi.org/ 10.1016/j.rcim.2010.05.008

Chakrabarti, A. et al. (2011), "Computer-based design synthesis research: an overview", Journal of Computing and Information Science in Engineering, Vol. 11 No. 2, p. 021003. https://doi.org/10.1115/1.3593409

Cho, J. et al. (2016), "KBE-PLM integration schema for engineering knowledge re-use and design automation", Proceedings of the IFIP International Conference on Product Lifecycle Management, Springer, Cham, pp. 43-55. https://doi.org/10.1007/978-3-319-54660-5_5

Cui, J. and Tang, M. (2017), "Towards generative systems for supporting product design", International Journal of Design Engineering, Vol. 7 No. 1, pp. 1-16. https://doi.org/10.1504/IJDE.2017.085639

Demminger, C. et al. (2016), "The Concept of Technical Inheritance in Operation: Analysis of the Information Flow in the Life Cycle of Smart Products", Procedia Technology, Vol. 26, pp. 79-88. https://doi.org/10.1016/j.protcy.2016.08.012 
Gembarski, P., Li, H. and Lachmayer, R. (2017), "Template-Based Modelling of Structural Components", International Journal of Mechanical Engineering and Robotics Research, Vol. 6 No. 5, pp. 336-342. https://doi.org/10.18178/ijmerr.6.5.336-342

Gembarski, P. (2018), Komplexitätsmanagement mittels wissensbasiertem CAD, TEWISS-Verlag, Garbsen.

Gips, J. (1999), "Computer implementation of shape grammars", NSF/MIT workshop on shape computation, Massachusetts Institute of Technology, Cambridge, MA.

Helms, B. (2013), Object-oriented graph grammars for computational design synthesis, [PhD Thesis], Technische Universität München.

Hirz, M. et al. (2013), Integrated computer-aided design in automotive development, Springer, Graz. https://doi.org/10.1007/978-3-642-11940-8

Hoisl, F. (2012), Visual, interactive 3D spatial grammars in CAD for computational design synthesis, [PhD Thesis], Technische Universität München.

Hoffmann, C. (2005), "Constraint-based computer-aided design", Journal of Computing and Information Science in Engineering, Vol. 5 No. 3, pp. 182-187. https://doi:10.1115/1.1979508

Kim, B. and Han, S. (2007), "Integration of history-based parametric translators using the automation APIs", International Journal of Product Lifecycle Management, Vol. 2 No. 1, pp. 18-29. https://doi.org/10. 1504/IJPLM.2007.012872

Krish, S. (2011), “A practical generative design method”, Computer Aided Design, Vol. 43 No. 1, pp. 88-100. https://doi.org/10.1016/j.cad.2010.09.009

Lachmayer, R. et al. (2017), "Algorithmic design evolution based on product life cycle information", CyberPhysical and Gentelligent Systems in Manufacturing and Life Cycle: Genetics and Intelligence-Keys to Industry 4.0, pp. 415-437. https://doi.org/10.1016/B978-0-12-811939-6.00004-2

La Rocca, G. (2011), Knowledge based engineering: Techniques to support aircraft design and optimization, [PhD Thesis], TU Delft.

Leinweber, S. (2014), "Wissensmanagement in der Produktentwicklung am Beispiel von Autotüren”, In: Tecklenburg, G. (Ed.), Karosseriebautage Hamburg, Springer, Wiesbaden, pp. 71-87. https://doi.org/10. 1007/978-3-658-05980-4_9

Li, H. and Lachmayer, R. (2019), "Automated Exploration of Design Solution Space Applying the Generative Design Approach", Proceedings of the Design Society: International Conference on Engineering Design, Cambridge University Press, Vol. 1, No. 1, pp. 1085-1094. https://doi.org/10.1017/dsi.2019.114

Lin, Y.S. et al. (2009), "A method and software tool for automated gearbox synthesis", ASME 2009 International Design Engineering Technical Conferences and Computers and Information in Engineering Conference, American Society of Mechanical Engineers, pp. 111-121. https://doi.org/10.1115/DETC2009-86935

Morello, L. et al. (2011), The Automotive Body: Volume I: Components Design, Springer Science \& Business Media. https://doi.org/10.1007/978-94-007-0513-5

Mozgova, I., Lachmayer, R. and Gottwald, P. (2015), "Formulations of Paradigms of Technical Inheritance", DS 80-8 Proceedings of the 20th International Conference on Engineering Design (ICED 15), Innovation and Creativity, Milan, Italy, Vol. 8, pp. 271-278.

Pahl, G. et al. (2013), Engineering design: a systematic approach, Springer Science \& Business Media. https://doi.org/10.1007/978-1-84628-319-2

Park, K. and Holt, N. (2010), "Parametric design process of a complex building in practice using programmed code as master model", International Journal of Architectural Computing, Vol. 8 No. 3, pp. 359-376. https://doi.org/10.1260/1478-0771.8.3.359

Sauthoff, B. (2017), Generative Parametrische Modellierung von Strukturkomponenten für die Technische Vererbung, TEWISS-Verlag, Garbsen.

Stokes, M. (2001), Managing Engineering Knowledge - MOKA: Methodology for Knowledge Based Engineering Applications, Professional Engineering Publishing Limited, London.

Tabrizi, B. and Walleigh, R. (1997), "Defining next-generation products: An inside look", Harvard Business Review, Vol. 75 No. 6, pp. 116-125.

Vajna, S. et al. (2009), CAx für Ingenieure: eine praxisbezogene Einführung, Springer Berlin Heidelberg. https://doi.org/10.1007/978-3-540-36039-1

VDI 5610-2 (2017), Knowledge management for engineering: knowledge-based engineering, Beuth Verlag, Berlin.

Verhagen, W. et al. (2012), "A critical review of Knowledge-Based Engineering: An identification of research challenges", Advanced Engineering Informatics, Vol. 26 No. 1, pp. 5-15. https://doi.org/10.1016/ j.aei.2011.06.004

Yin, C. and Ma, Y. (2012), "Parametric feature constraint modeling and mapping in product development", Advanced Engineering Informatics, Vol. 26 No. 3, pp. 539-552. https://doi.org/10.1016/j.aei.2012.02.010 